\title{
Giant bilateral adrenal myelolipomas in two Chinese families with congenital adrenal hyperplasia
}

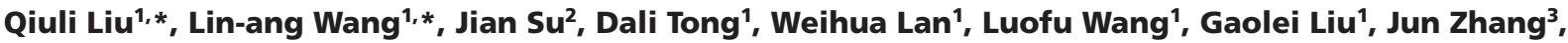 \\ Victor Wei Zhang ${ }^{4,5}$, Dianzheng Zhang, ${ }^{6}$ Rongrong Chen7, Qingyi Zhu² and Jun Jiang ${ }^{1}$
}

'Department of Urology, Institute of Surgery Research, Daping Hospital, Third Military Medical University, Chongqing, People's Republic of China ${ }^{2}$ Department of Urology, Affiliated Hospital of Nanjing University of Traditional Chinese Medical, Nanjing, People's Republic of China ${ }^{3}$ Department of Obstetrics, Third Affiliated Hospital of Sun Yat-sen University, Guangzhou, People's Republic of China ${ }^{4}$ Department of Molecular and Human Genetics, Baylor College of Medicine, Houston, Texas, USA

${ }^{5}$ AmCare Genomics Lab, Guangzhou, People's Republic of China

${ }^{6}$ Department of Bio-Medical Sciences, Philadelphia College of Osteopathic Medicine, Philadelphia, Pennsylvania, USA

${ }^{7}$ Geneplus-Beijing Institute, Beijing, People's Republic of China

Correspondence should be addressed to J Jiang or Q Zhu: jiangjun_64@163.com or zhuqy1971@126.com

*(Q Liu and L Wang contributed equally to this work)

\begin{abstract}
Congenital adrenal hyperplasia $(\mathrm{CAH})$ is one of the most prevalent, and potentially severe, genetic inborn errors of steroid synthesis directly affecting metabolism. Most patients are diagnosed and treated at an early age. There have been very limited reports of adults with $\mathrm{CAH}$-associated adrenal myelolipomas. We aimed to analyze two families with CAH-associated giant adrenal myelolipomas caused by defects in CYP21A2 and CYP17A1 genes. A total of 14 individuals from two unrelated families were identified with either CYP21A2 or CYP17A1 mutations. Of note, five patients were found with adrenal myelolipomas. Total DNA isolated from the peripheral blood of the two probands was screened for potential mutations in the following susceptibility genes of CAH: CYP21A2, CYP11B1, CYP17A1, HSD17B3, HSD3B2, ARMC5, and STAR using target capture-based deep sequencing; and Sanger sequencing was conducted for the family members to detect the potential mutations. The following results were obtained. In family 1 , molecular genetics sequencing revealed a compound heterozygous mutation (c.293-13C>G/c.518T>A, p.I173N) in CYP12A2 in the patient and his brother. In family 2, all three female patients with adrenal myelolipomas were found to have a compound heterozygous mutation (c.1118A>T, p.H373L/c.1459_1467del9, p.D487_F489del) in CYP17A1. To avoid giant CAH-associated adrenal myelolipomas in adults, it is important to identify $\mathrm{CAH}$ early so that appropriate treatment can be initiated to interrupt the chronic adrenal hyperstimulation resulting from increased ACTH. Genetic testing and counseling could be useful in $\mathrm{CAH}$.
\end{abstract}

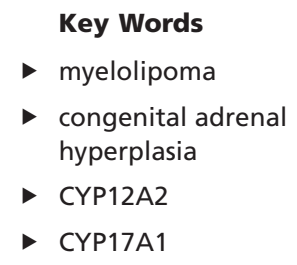

Endocrine Connections (2018) 7, 1136-1141

\section{Introduction}

Congenital adrenal hyperplasia $(\mathrm{CAH})$ is one of the most severe disorders of metabolism with an overall incidence of approximately 1:15,000 worldwide (1). CAH encompasses a group of enzymatic deficiencies of cortisol synthesis, inherited in an autosomal recessive manner. Defective cortisol production results in negative feedback at the hypothalamus and pituitary gland, leading to excessive secretion of corticotrophin-releasing hormone

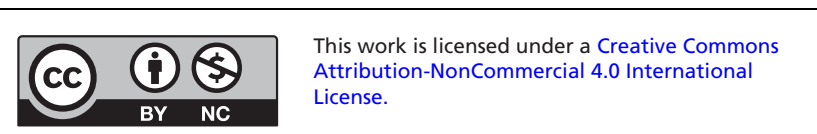


and adrenocorticotropic hormone (ACTH), respectively. Increased levels of ACTH are not only unable to compensate for the deficient cortisol synthesis, but their trophic effects also produce adrenal gland enlargement (2).

Pathologically, CAH is mainly caused by an inborn error of steroid synthesis. Six cytochrome P450 (CYP) enzymes including CYP11A1, CYP11B1, CYP11B2, CYP17A1, CYP19A1 and CYP21A2 are involved in the synthesis of steroid hormones. Although deficiencies of any of these enzymes can result in CAH (3), CYP21A2 deficiency (21OHD) accounts for up to 95\% of all CAH cases (4). The CYP21A2 gene is located on $6 \mathrm{p} 21.3$, approximately $30 \mathrm{~kb}$ from its pseudogene (CYP21A1P). CYP21A2 and CYP21A1P share approximately 98\% homology in their exons and 96\% homology in their introns; therefore, a high frequency of recombination events may occur between the gene and pseudogene, leading to varying degrees of CYP21A2 enzymatic deficiency. Complete deficiency of CYP21A2 activity leads to both glucocorticoid and mineralocorticoid deficiencies, as well as severe adrenal-derived androgen excess, resulting in the virialized external genitalia of newborn girls. Compared to CYP21A2-related disorders, CYP17A1 deficiency is rare. The CYP17 gene is located on chromosome 10q24-q25 and encodes $17 \alpha$-hydroxylase, which catalyzes the $17 \alpha$-hydroxylation of pregnenolone and progesterone and the 17,20-cleavage of the corresponding hydroxylated steroids (5). CYP17A1 deficiency leads to the impaired synthesis of both glucocorticoids and androgen, resulting in increased ACTH secretion. Patients present with hypertension, hypokalemia and hypogonadism. Both 46, XY and 46, XX individuals have feminine external genitalia and present with amenorrhea and absent secondary sexual characteristics during puberty (2).

Adrenal myelolipomas are rare, benign and endocrinologically inactive neoplasms composed of mature fat tissue and mixed myeloid and erythroid tissue (6). They account for approximately $8 \%$ of adrenal incidentalomas (6). In most cases, adrenal myelolipomas are unilateral and rarely exceed $4 \mathrm{~cm}$. However, very large and bilateral myelolipomas have been reported, especially in the setting of $\mathrm{CAH}$ (7). Although these tumors are benign, huge masses can produce symptoms such as flank pain and abdominal discomfort because of rupture, hemorrhage or necrosis (7). Some patients with giant adrenal myelolipomas may present with even more severe symptoms, including hematuria, renovascular hypertension and surgical emergencies such as retroperitoneal hemorrhage (8). CAH and Cushing's syndrome are two of the most common causes of adrenal myelolipomas (9), and $4 \%$ of patients with $\mathrm{CAH}$ will develop a myelolipoma (10). In this report, we describe two adult families with $\mathrm{CAH}$-associated giant adrenal myelolipomas secondary to compound heterozygous mutations in CYP21A2 and CYP17A1 genes.

\section{Materials and methods}

\section{Patients}

A total of 15 individuals from two unrelated families were sequenced to evaluate whether they carried the mutations or not. Among them, 14 individuals were identified with either CYP21A2 or CYP17A1 mutations. Of note, five patients were found with adrenal myelolipomas. The medical records including CT or MRI scan and laboratory /pathological examinations, as well as gene sequencing results were collected and analyzed. Additional information regarding their family history was obtained by further interviewing.

\section{Compliance with ethical standards}

All procedures involving human participants were carried out in accordance with the ethical standards of the institutional research committee and with the 1964 Helsinki Declaration and its later amendments or comparable ethical standards. The Daping Hospital of Third Military Medical University waived institutional review board approval for the study; however, written informed consent for the use of medical records and related images was obtained from the patients.

\section{Genetic analysis}

Total DNA isolated from the peripheral blood of the two probands was screened for potential mutations in the following susceptibility genes of CAH: CYP21A2, CYP11B1, CYP17A1, HSD17B3, HSD3B2, ARMC5 and STAR using Target Capture-Based Deep Sequencing (AmCare Genomic Laboratory, Guangzhou, Guangdong, China). Briefly, the process included fragmentation and construction of a genomic DNA, hybridization with custom made probe library (Nimblegen, Roche) to capture the genes of interested. The sequencing was carried out on NGS platform (HiSeq X system; Illumina) with PEx150 read length according to the manufactory's specification. The bioinformatics analysis was performed with in-house pipeline to identify rare or novel variants with Gnomad, HMGD, ClinVar, dbSNP for filtering and computational prediction algorithms,

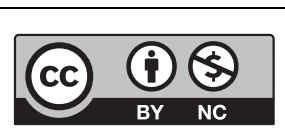

This work is licensed under a Creative Commons Attribution-NonCommercial 4.0 International License. 
PolyPhen-2, SIFT and MutationTaster, for pathogenesis evaluation. After the identification of the variants in the proband, family co-segregation analyses were performed using Sanger sequencing. The corresponding primers were designed by Primer 3.0 and available upon request. The final pathogenicity of the variants was estimated using the American College of Medical Genetics and Genomics guidelines.

\section{Results}

\section{Clinical features of the patients}

\section{Family 1}

A 59-year-old man was referred to our hospital in April 2012 with abdominal pain. CT revealed low attenuated masses in both adrenal glands (Fig. 1A). On admission, his blood pressure was $132 / 78 \mathrm{mmHg}$ and heart rate was 78 beats/min. His physical examination was unremarkable, revealing no signs of Cushing's syndrome, hyperaldosteronism or pheochromocytoma. Laboratory results demonstrated elevated levels of 17-hydroxycorticosteroid $(17-\mathrm{OH} ; 38.5 \mu \mathrm{mol} / 24 \mathrm{~h}$, normal $8.3-33.2 \mu \mathrm{mol} / 24 \mathrm{~h})$ and 17-ketosteroid (7-KS; $98.8 \mu \mathrm{mol} / 24 \mathrm{~h}$, normal $20.8-76.3 \mu \mathrm{mol} / 24 \mathrm{~h}$ ).
On April 26, 2012, the patient underwent open bilateral adrenalectomies. Intraoperative findings revealed a $1.56 \mathrm{~kg}$ mass in the left adrenal and a $3.05 \mathrm{~kg}$ mass in the right adrenal (Fig. 1A, right lower corner). Pathology results confirmed the presence of bilateral adrenal myelolipomas (Fig. 1C and D). The patient's older brother had previously undergone bilateral adrenalectomies in 1989 at the age of 50 years for pathology confirmed adrenal myelolipomas. Their parents died early with unknown cause. The other family members were screened for the disease by CT or MRI, as well as hormonal testing. No abnormality was found.

\section{Family 2}

A 36-year-old woman presented to the affiliated hospital of Nanjing University of Traditional Chinese Medical with fatigue, headaches and asyndesis (unspeakable) in March 2012. She had hypokalemia since childhood and hypertension for the past 6 years. On admission, her blood pressure was $170 / 110 \mathrm{mmHg}$. MRI revealed bilateral adrenal masses with a signal intensity similar to that of fat, suggesting the presence of adrenal myelolipomas (Fig. 1B). Laboratory results showed increased ACTH (more than $1250 \mathrm{pg} / \mathrm{mL}$ ) and decreased cortisol (less than $2 \mu \mathrm{g} / \mathrm{dL}$ ). In April and November 2012, the patient
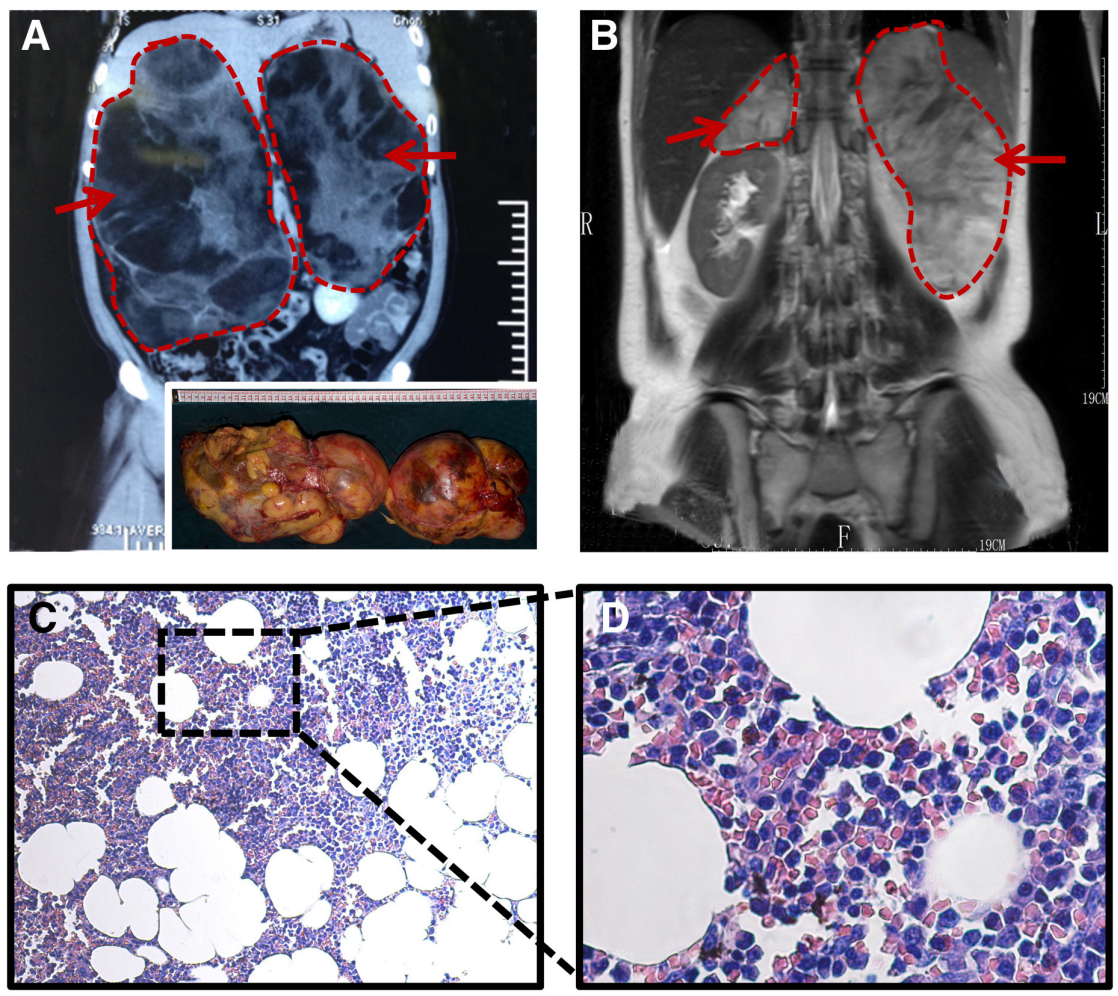

Figure 1

Representative CT scan, MRI scan and hematoxylin and eosin ( $H \& E$ ) staining of the presenting patients (probands) in each family. (A) CT and MRI images showing bilateral adrenal masses in the proband for family 1 . The adrenals removed during surgery are shown in the right lower corner. (B) MRI scan showing bilateral adrenal masses in the proband for family 2 . ( $C$ and D) $H$ \& $E$ staining of the masses in the proband for family 1. Magnification: $\times 100$ (C), $\times 400$ (D).

https://ec.bioscientifica.com https://doi.org/10.1530/EC-18-0273 (c) 2018 The authors Published by Bioscientifica Ltd

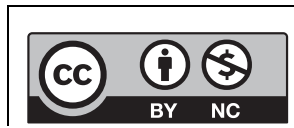

This work is licensed under a Creative Commons Attribution-NonCommercial 4.0 International License. 
underwent left and right adrenalectomies, respectively. Intraoperative findings showed a $20 \mathrm{~cm} \times 15 \mathrm{~cm} \times 10 \mathrm{~cm}$ mass in the left adrenal and another smaller mass in the right adrenal.

In June 2012, the patient's 32-year-old sister was referred to the affiliated hospital of Nanjing University of Traditional Chinese Medical because of increased fatigue. She had presented with fatigue 8 years before, at which time she was found to have hypokalemia. CT scan revealed a mass in her left adrenal. Laboratory results showed increased ACTH $(271 \mathrm{pg} / \mathrm{mL})$ and decreased cortisol (less than $2 \mu \mathrm{g} / \mathrm{dL}$ ). In October 2012, she underwent left adrenalectomy, and the pathology results revealed an adrenal myelolipoma.

In July 2012, the patient's 37-year-old sister was also referred to the affiliated hospital of Nanjing University of Traditional Chinese Medical. She had hypertension and hypokalemia for the previous 3 years. CT scan revealed bilateral adrenal masses. Laboratory results showed increased ACTH $(503 \mathrm{pg} / \mathrm{mL})$ and decreased cortisol $(3.1 \mu \mathrm{g} / \mathrm{dL})$. In July and December 2012, she underwent left and right adrenalectomies, respectively. Pathology results confirmed the presence of adrenal myelolipomas. The other family members were asymptomatic on CT or MRI.

\section{Identification of the mutations}

\section{Family 1}

Considering the positive family history, CAH was suspected and genomic analysis was performed using DNA isolated from peripheral blood leukocytes. A compound heterozygous mutation, consisting of c.293-13C>G and c.518T >A, p.I173N, was identified in the CYP12A2 gene (Fig. 2A). Sanger sequencing of the CYP12A2 gene was conducted in the family members. The brother had the same compound heterozygous mutation as the patient. The patient's sister exhibited only the c.518T >A, p.I173N mutation. Two of the patient's daughters had only the c.293-13C>G mutation, while another daughter and her daughter had only the c.518T $>$ A, p.I173N mutation. The daughter of the patient's brother carried only the c.293-13C>G mutation. The family pedigree is shown in Fig. 2C.

\section{Family 2}

Considering the positive family history, CAH was suspected. Genomic sequencing of the patient revealed a compound heterozygous mutation, consisting of c.1118A $>$ T, p.H373L, and c.1459_1467del9, p.D487_F489del in the CYP17A1 gene (Fig. 2B). Sanger sequencing was conducted on the family
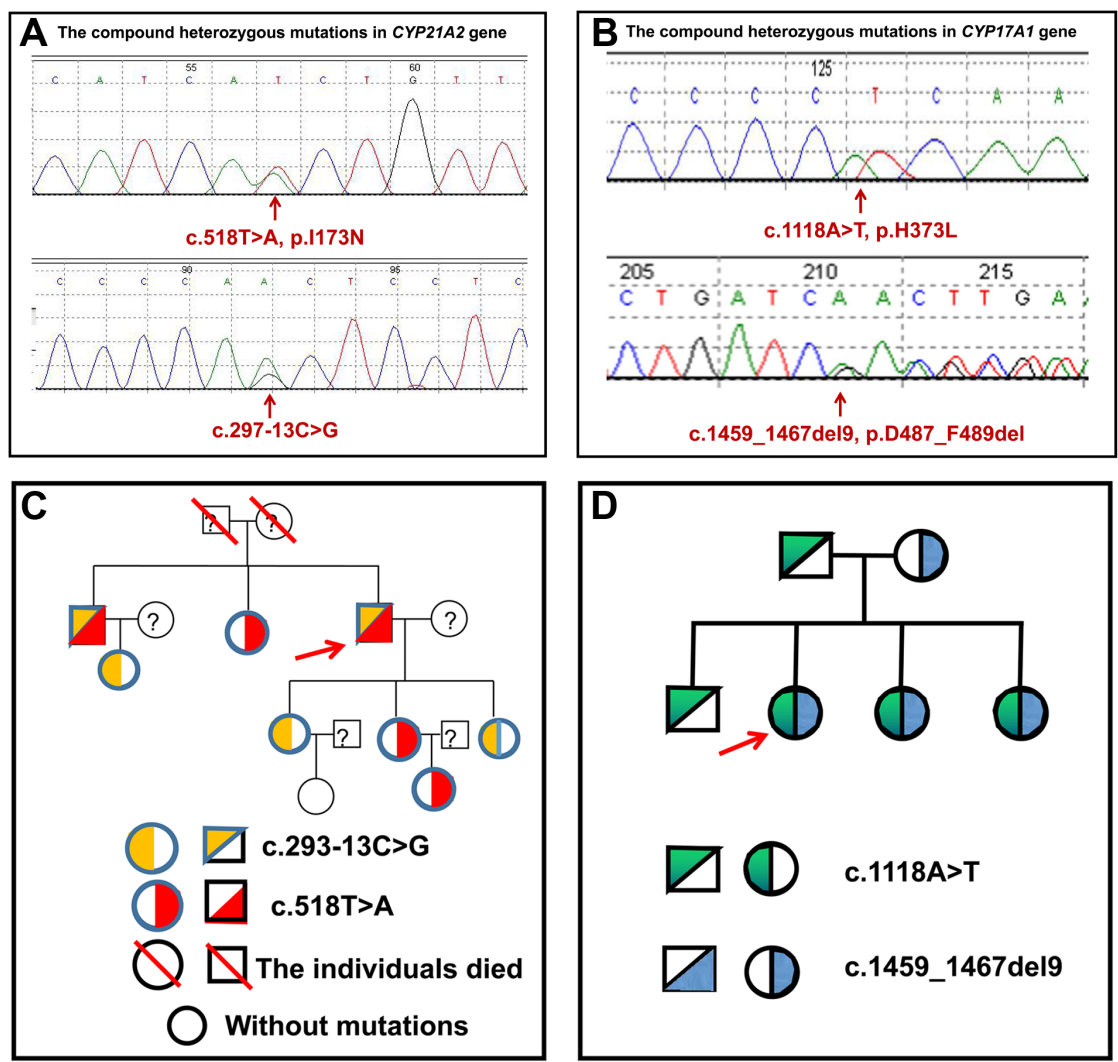

Figure 2

Gene sequencing data and family pedigrees. ( $A$ and $B$ ) Compound heterozygous mutations in CYP21A2 gene in family 1 (A) and in CYP17A1 gene in family 2 (B). ( $C$ and $D)$ Pedigrees for families 1 (C) and 2 (D). The red arrow indicates the proband. '?' represents that the patients were not conducted with gene sequencing. https://ec.bioscientifica.com

https://doi.org/10.1530/EC-18-0273 (c) 2018 The authors Published by Bioscientifica Ltd

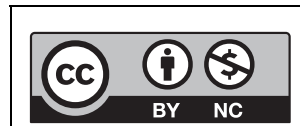

This work is licensed under a Creative Commons Attribution-NonCommercial 4.0 International License. 
members, and the patient's two sisters were found to have the same compound heterozygous mutation. Her father and asymptomatic brother carried only the c.1118A $>$ T, p.H373L mutation, and her mother had only the c.1459_1467del9, p.D487_F489del mutation. The family pedigree is shown in Fig. 2D.

\section{Discussion}

A few cases of CAH-associated adrenal myelolipoma have been reported previously with most of them caused by $21-\mathrm{OH}$ deficiency. The second most common cause was $17-\mathrm{OH}$ deficiency (11). In the current report, two patients in family 1 presented with CAH-associated giant adrenal myelolipomas secondary to $21-\mathrm{OH}$ deficiency caused by a compound heterozygous mutation (c.293-13C>G/c.518T>A, p.I173N). Three patients in family 2 presented with $\mathrm{CAH}$-associated adrenal myelolipomas because of $17-\mathrm{OH}$ deficiency resulting from a compound heterozygous mutation (c.1118A $>\mathrm{T}$, p.H373L/c.1459_1467del9, p.D487_F489del). All the four mutations have been reported to be associated with $\mathrm{CAH}$, respectively $(12,13,14,15)$. However, no adrenal myelolipoma was found with single mutations. Here, we firstly described these two compound heterozygous mutations in two unrelated families and found that only these compound heterozygous mutations, but not the single mutation, in the CYP genes can result in adrenal myelolipomas.

A previous study reported that approximately $65-75 \%$ of $\mathrm{CAH}$ patients had compound heterozygous mutations, and the phenotype correlated with the less severely mutated allele (16). However, our results suggest that CAH-associated adrenal myelolipomas occurred only when compound heterozygous mutations were present. In addition, the adrenal myelolipomas caused by 21-OH deficiency seemed to occur at an older age than those caused by 17-OH deficiency, as the individuals in family 1 were diagnosed with adrenal myelolipomas at approximately 50-60 years of age, whereas those in family 2 were diagnosed at 30-40 years of age. A previous report noted that most adrenal myelolipomas due to $17-\mathrm{OH}$ deficiency were unilateral (11). However, the mutations (H373L/D487_F489del) in family 2 tended to produce bilateral adrenal myelolipomas; the youngest sister presented with a tumor that was unilateral, but this may have been because of her younger age.

Notably, chronic ACTH hyperstimulation is thought to trigger metaplasia in $\mathrm{CAH}$-associated adrenal myelolipomas, as more myelolipomas have been described in the setting of ACTH-excess CAH (17). Although benign, CAHassociated adrenal myelolipomas may grow rapidly when exposed to chronically elevated ACTH levels for extended period (18). Most patients in previous reports were either untreated or had stopped taking their CAH medications for an extended period (19). The patients in our report were all untreated for a prolonged period of time, which led to chronic ACTH hyperstimulation and subsequent adrenal myelolipomas. Therefore, to avoid giant $\mathrm{CAH}$-associated adrenal myelolipomas, it is important to identify $\mathrm{CAH}$ as early as possible to begin appropriate treatment and, thereby, interrupt the chronic adrenal hyperstimulation resulting from increased ACTH levels.

Although adrenal myelolipomas were seen in both families with different phenotypes. For example, hypertension and hypokalemia were observed in only family 2 . The divergent phenotypes reflected the presence of mutations in different genes, CYP21A2 and CYP17A1. CYP21A2 deficiency produces reduced aldosterone and cortisol and excess testosterone, whereas CYP17A1 deficiency leads to decreased cortisol and testosterone and increased aldosterone, which causes hypertension and hypokalemia (2). In the same family, members with concurrent mutations in both alleles presented with adrenal myelolipomas, whereas individuals with either mutation in just one allele did not. This difference in tumor occurrence may be due to varying degrees of enzymatic deficiency. A previous study found that patients with severe mutations causing complete deficiency of enzymatic activity presented with severe phenotypes, whereas patients with mild mutations exhibited the nonclassical form of CAH (20). In vitro expression analysis demonstrated that complete deficiency of 21-hydroxylase enzymatic activity could result from multiple mutations in the CYP21A2 gene, such as large deletion $\triangle 707-714$, Q318X and R356W mutations. These mutations could cause severe phenotypes, whereas other mutations, including P30L (89 C to T), V281L (1685 G to T) and P453S (2580 C to T) mutations, may lead to 20-60\% residual enzymatic activity, which is likely to produce a moderate phenotype (20). Furthermore, a previous study reported that patients with the classical form of CAH most commonly had an IVS2 AS-13 (A/C to G) mutation and a large deletion in the CYP21A2 gene (20). These results indicate that a strong genotype-phenotype correlation exists in CAH. Therefore, individuals with the compound mutations described in the current report should be treated early to avoid chronic ACTH hyperstimulation and actively monitored for adrenal myelolipomas.

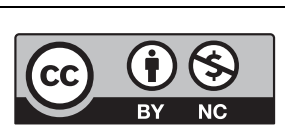

This work is licensed under a Creative Commons Attribution-NonCommercial 4.0 International License. 
This highlights the importance of genetic testing and counseling in $\mathrm{CAH}$-associated adrenal myelolipomas.

In conclusion, we found that compound heterozygous mutations, but not the single mutation, in the CYP genes can result in adrenal myelolipomas. Our finding also suggests that early diagnosis and timely treatment for chronic adrenal hyperstimulation may prevent the development of giant CAH-associated adrenal myelolipomas. In addition, genetic testing and counseling could be valuable means in dealing with $\mathrm{CAH}$.

\section{Declaration of interest}

The authors declare that there is no conflict of interest that could be perceived as prejudicing the impartiality of the research reported.

\section{Funding}

This work did not receive any specific grant from any funding agency in the public, commercial, or not-for-profit sector.

\section{Acknowledgement}

The authors would like to thank all patients and families in this study for their collaboration.

\section{References}

1 Miller WL \& Auchus RJ. The molecular biology, biochemistry, and physiology of human steroidogenesis and its disorders. Endocrine Reviews 201132 81-151. (https://doi.org/10.1210/er.2010-0013)

2 Turcu AF \& Auchus RJ. The next 150 years of congenital adrenal hyperplasia. Journal of Steroid Biochemistry and Molecular Biology 2015 153 63-71. (https://doi.org/10.1016/j.jsbmb.2015.05.013)

3 Zhao B, Lei L, Kagawa N, Sundaramoorthy M, Banerjee S, Nagy LD, Guengerich FP \& Waterman MR. Three-dimensional structure of steroid 21-hydroxylase (cytochrome P450 21A2) with two substrates reveals locations of disease-associated variants. Journal of Biological Chemistry 2012287 10613-10622. (https://doi.org/10.1074/jbc. M111.323501)

4 White PC \& Speiser PW. Congenital adrenal hyperplasia due to 21-hydroxylase deficiency. Endocrine Reviews 200021 245-291. (https://doi.org/10.1210/edrv.21.3.0398)

5 Zuber MX, Simpson ER \& Waterman MR. Expression of bovine 17 alphahydroxylase cytochrome P-450 cDNA in nonsteroidogenic (COS 1) cells. Science 1986234 1258-1261. (https://doi.org/10.1126/science.3535074)

6 Mansmann G, Lau J, Balk E, Rothberg M, Miyachi Y \& Bornstein SR. The clinically inapparent adrenal mass: update in diagnosis and management. Endocrine Reviews 200425 309-340. (https://doi. org/10.1210/er.2002-0031)

7 Ioannidis O, Papaemmanouil S, Chatzopoulos S, Paraskevas G, Konstantara A, Kotronis A, Kakoutis E \& Makrantonakis A. Giant bilateral symptomatic adrenal myelolipomas associated with congenital adrenal hyperplasia. Pathology Oncology Research 201117 775-778. (https://doi.org/10.1007/s12253-010-9330-2)
8 Brogna A, Scalisi G, Ferrara R \& Bucceri AM. Giant secreting adrenal myelolipoma in a man: a case report. Journal of Medical Case Reports 20115 298. (https://doi.org/10.1186/1752-1947-5-298)

9 Cha JS, Shin YS, Kim MK \& Kim HJ. Myelolipomas of both adrenal glands. Korean Journal of Urology 201152 582-585. (https://doi. org/10.4111/kju.2011.52.8.582)

10 Nermoen I, Rorvik J, Holmedal SH, Hykkerud DL, Fougner KJ, Svartberg J, Husebye ES \& Lovas K. High frequency of adrenal myelolipomas and testicular adrenal rest tumours in adult Norwegian patients with classical congenital adrenal hyperplasia because of 21-hydroxylase deficiency. Clinical Endocrinology 201175 753-759. (https://doi.org/10.1111/j.1365-2265.2011.04151.x)

11 Mermejo LM, Elias Junior J, Saggioro FP, Tucci Junior S, Castro M, Moreira AC \& Elias PC. Giant adrenal myelolipoma associated with 21-hydroxylase deficiency: unusual association mimicking an androgen-secreting adrenocortical carcinoma. Arquivos Brasileiros de Endocrinologia e Metabologia 201054 419-424. (https://doi. org/10.1590/S0004-27302010000400012)

12 Friaes A, Rego AT, Aragues JM, Moura LF, Mirante A, Mascarenhas MR, Kay TT, Lopes LA, Rodrigues JC, Guerra S, et al. CYP21A2 mutations in Portuguese patients with congenital adrenal hyperplasia: identification of two novel mutations and characterization of four different partial gene conversions. Molecular Genetics and Metabolism 200688 58-65. (https://doi.org/10.1016/j.ymgme.2005.11.015)

13 Toraman B, Okten A, Kalay E, Karaguzel G, Dincer T, Acikgoz EG \& Karaguzel A. Investigation of CYP21A2 mutations in Turkish patients with 21-hydroxylase deficiency and a novel founder mutation. Gene 2013513 202-208. (https://doi.org/10.1016/j.gene.2012.10.059)

14 Monno S, Ogawa H, Date T, Fujioka M, Miller WL \& Kobayashi M. Mutation of histidine 373 to leucine in cytochrome P450c17 causes 17 alpha-hydroxylase deficiency. Journal of Biological Chemistry 1993 268 25811-25817.

15 Fardella CE, Zhang LH, Mahachoklertwattana P, Lin D \& Miller WL. Deletion of amino acids Asp487-Ser488-Phe489 in human cytochrome P450c17 causes severe 17 alpha-hydroxylase deficiency. Journal of Clinical Endocrinology and Metabolism 199377 489-493. (https://doi.org/10.1210/jcem.77.2.8345056)

16 Jaaskelainen J, Levo A, Voutilainen R \& Partanen J. Population-wide evaluation of disease manifestation in relation to molecular genotype in steroid 21-hydroxylase (CYP21) deficiency: good correlation in a well defined population. Journal of Clinical Endocrinology and Metabolism 199782 3293-3297. (https://doi.org/10.1210/ jcem.82.10.4271)

17 Hagiwara H, Usui T, Kimura T, Tagami T, Naruse M, Minamiguchi S, Kato T, Okuno H \& Shimatsu A. Lack of ACTH and androgen receptor expression in a giant adrenal myelolipoma associated with 21-hydroxylase deficiency. Endocrine Pathology 200819 122-127. (https://doi.org/10.1007/s12022-008-9034-2)

18 Miyazaki Y, Yoshida M, Doi J. A case of adrenal myelolipoma associated with adrenogenital syndrome. Hinyokika Kiyo. Acta Urologica Japonica 199036 35-39.

19 Murakami C, Ishibashi M, Kondo M, Ohshiro S, Fujita M, Sato S, Kako M, Furue H, Mizuguchi K \& Yamaji T. Adrenal myelolipoma associated with congenital adrenal 21-hydroxylase deficiency. Internal Medicine 199231 803-806. (https://doi.org/10.2169/ internalmedicine.31.803)

20 Wilson RC, Nimkarn S, Dumic M, Obeid J, Azar MR, Najmabadi H, Saffari F \& New MI. Ethnic-specific distribution of mutations in 716 patients with congenital adrenal hyperplasia owing to 21-hydroxylase deficiency. Molecular Genetics and Metabolism 200790 414-421. (https://doi.org/10.1016/j.ymgme.2006.12.005)

Received in final form 2 September 2018 Accepted 5 September 2018

Accepted Preprint published online 7 September 2018

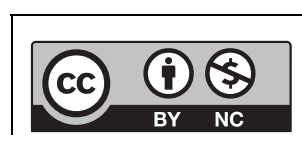

This work is licensed under a Creative Commons Attribution-NonCommercial 4.0 International License. 